\title{
EL CONCEPTO DE SOSTENIBILIDAD EN AGROECOLOGÍA
}

\section{THE CONCEPT OF SUSTAINABILITY IN AGROECOLOGY}

\author{
Luis Fernando Gómez ${ }^{1}$, Leonardo Alberto Ríos-Osorio ${ }^{2}$, María Luisa Eschenhagen Durán ${ }^{3}$
}

\begin{abstract}
${ }^{1}$ Ingeniero Químico, M.Sc., Departamento de Ciencias Sociales y Humanas. Universidad de Ciencias Aplicadas y Ambientales U.D.C.A, calle 222 No 54-37, Bogotá, D.C., Colombia, e-mail: luis.fgomez@udca.edu.co; ${ }^{2}$ Bacteriólogo, Ph.D., Escuela de Microbiología. Universidad de Antioquia, Medellín-Colombia, e-mail: leonardo.rios@udea.edu.co; ${ }^{3}$ Licenciada en Ciencias Sociales, Ph.D., Escuela de Ciencias Sociales. Universidad Pontificia Bolivariana, Medellín-Colombia, e-mail: marialuisa.eschenhagen@upb.edu.co
\end{abstract}

Rev. U.D.C.A Act. \& Div. Cient. 18(2): 329-337, Julio-Diciembre, 2015

\section{RESUMEN}

Dentro de la teoría de las ciencias ambientales, se ha dicho que ellas tienen como propósito lograr la sostenibilidad de diferentes sistemas de referencia. El objetivo de la presente investigación es determinar los planteamientos que existen alrededor de la sostenibilidad y su lugar dentro de la teoría agroecológica. Se realizó una revisión sistemática en bases de datos científicas y se complementó con revistas especializadas y libros del campo de la agroecología. Se encontró, que la agroecología plantea como objetivo la sostenibilidad, pero no se presenta claridad en el sistema de referencia, proponiendo los agroecosistemas, los sistemas alimentarios, la agricultura, el desarrollo y el desarrollo rural.

Palabras clave: Agricultura sustentable, agroecosistemas, desarrollo rural, soberanía alimentaria.

\section{SUMMARY}

Scholars have established sustainability as the goal of environmental sciences. The objective of this research was to establish definitions regarding the concept of sustainability and its place within agroecology theory. For that, a systematic review was conducted in global scientific databases, complemented with specialized journals and books. It was found that there is no agreement on the system of reference, so sustainable agriculture, development, rural development, as well as sustainability of agroecosystems, and of food systems are proposed as goals of agroecology. Also, there is no consensus on the kind of sustainability agroecology embraces, but integral sustainability has a broader acceptance.

Key words: Sustainable agriculture, agroecosystems, rural development, food sovereignty.

\section{INTRODUCCIÓN}

Desde su planteamiento, como desarrollo sostenible en el Informe Brundtland, en 1987, el concepto de sostenibilidad ha adquirido un papel central dentro de las ciencias ambientales, hasta el punto de ser considerado el principio de acción teleológico de éstas (Dilworth, 2009), lo que significa, que el conocimiento y la comprensión -obtenidos en este tipo de ciencias- es visto como un medio para lograr la sostenibilidad.

A pesar de ello, las revisiones bibliográficas que han buscado caracterizar a la agroecología, es decir, la ciencia que estudia los sistemas de producción con seres vivos desde una perspectiva sistémica y transdisciplinaria, no se han detenido en este aspecto; por ejemplo, Wezel \& Soldat (2009), se centran en el objeto de estudio, mientras Tomich et al. (2011) analizan las diferentes disciplinas que confluyen en el campo de la agroecología, al igual que los problemas a futuro que ella debe enfrentar. Esto ha llevado a que aún no se presenten estudios sistemáticos ampliamente referenciados, que hagan un esfuerzo de establecer qué se entiende por sostenibilidad dentro de la agroecología y el papel que ella cumple dentro de esta ciencia; esto es relevante, porque aún no existe un consenso alrededor de lo que se debe entender por sostenibilidad.

Dentro de la actual discusión sobre la sostenibilidad, se pueden reconocer cuatro acepciones ampliamente utilizadas. En primer lugar, está la definición de desarrollo sostenible, que comprende la satisfacción de las necesidades actuales de la humanidad, sin comprometer la satisfacción de las necesidades de las generaciones futuras de la especie; segundo, está la sostenibilidad débil, que hace referencia al aumento o conservación del nivel actual del capital global, por lo que es una aproximación economicista a este concepto; en 
tercer lugar, se encuentra la sostenibilidad fuerte, en la que prima la sostenibilidad ecológica, es decir, que las relaciones de explotación de la biosfera no superen su capacidad de renovación y, por último, está la sostenibilidad integral, que promulga un equilibrio o niveles satisfactorios en las esferas económica, ecológica y social (Luffiego \& Rabadán, 2000).

Además, se ha señalado que es necesario articular el concepto de sostenibilidad a un sistema de referencia, pues es a partir de éste que cobra sentido. Así, no se habla de sostenibilidad en abstracto sino de construcciones sostenibles, ganadería sostenible, ciudades sostenibles, entre otros (RíosOsorio et al. 2013a).

Asimismo, Sikor \& Norgaard (1999) han advertido que las implicaciones de la sostenibilidad varían en función de si se aborda desde un marco científico convencional o uno constructivista; desde el primero, se considera que la sostenibilidad es un concepto objetivo, que se puede definir desde la ciencia, mientras que el enfoque constructivista, afirma que este concepto comprende juicios de valor, por lo que es necesario establecer con comunidades locales lo que se debe sostener, cómo y por quién. En consecuencia, se ha señalado que la reflexión conceptual de la sostenibilidad comprende dos aspectos: uno positivo y uno normativo; la sostenibilidad positiva establece qué se entiende por sostenibilidad dentro de una teoría, teniendo en cuenta que no existe una sola acepción del término, mientras el segundo, determina qué debe ser la sostenibilidad, señalando el sistema de referencia y los elementos que componen la sostenibilidad, al igual que la manera para alcanzarla (Ríos-Osorio et al. 2013a).

El objetivo de la presente revisión sistemática es determinar los planteamientos que existen alrededor de la sostenibilidad y su lugar dentro del discurso agroecológico, a nivel teórico. Para ello, se comienza con las definiciones generales de sostenibilidad, para luego pasar a los sistemas de referencia, que se han establecido dentro de la agroecología, como principio de acción teleológico u objetivo de ésta.

\section{MATERIALES Y MÉTODOS}

Se realizó una revisión sistemática de literatura en las bases de datos globales Science Direct, Jstor, Springer Link, Dialnet y Academic Search Complete, utilizando como término de búsqueda, agroecología; se consideraron elegibles los artículos que contenían el término de búsqueda en el título. A los artículos elegibles, se les aplicaron los siguientes criterios de inclusión, para su revisión completa: artículos publicados entre enero de 2005 y febrero de 2015; artículos publicados en idiomas inglés, español o portugués y artículos de reflexión o teóricos. A su vez, se descartaron aquellos artículos que no seguían la estructura IMRAD, propia de un artículo original, pero que se centraban en una región específica.
Los artículos hallados fueron complementados con artículos de reflexión, teóricos o resúmenes de ponencias, publicados en Revista Brasileira de Agroecología y Agroecología de la Universidad de Murcia -publicaciones especializadas-, con el fin de hacer más comprensiva la búsqueda, teniendo en cuenta, en la relevancia, que Brasil y Latinoamérica registran diversidad de publicaciones, en el campo de la agroecología, al igual que con libros, editados en el mismo período, de 2005 y febrero de 2015.

\section{RESULTADOS Y DISCUSIÓN}

Definición de sostenibilidad: Es claro, en los textos estudiados, que la agroecología tiene como objetivo la sostenibilidad, lo que concuerda con la caracterización de ciencias ambientales, de Dilworth (2009); sin embargo, los textos estudiados muestran que dentro de la agroecología no existe un consenso acerca de qué se debe entender por sostenibilidad ni desde dónde se debe aproximar. La agroecología, como muchos de los discursos académicos que han surgido a raíz de la crisis ecológica, ha cuestionado la epistemología de la ciencia convencional, lo que incluye su pretensión de universalidad y de neutralidad (Borsatto \& Carmo, 2012). En consecuencia, autores como Gutiérrez et al (2008), Warner (2008) y Silva \& De Biase (2012) disertan de la necesidad de un enfoque constructivista de la sostenibilidad, en el que se reconozca que ésta no responde a un criterio neutral que se pueda establecer a priori y para todos los casos, sino que debe ser contextual y producto de discusiones entre las diversas partes afectadas, por lo que no es posible dar una definición única desde la teoría; no obstante, en los textos estudiados son recurrentes definiciones canónicas de la sostenibilidad, como el desarrollo sostenible o la sostenibilidad integral, mientras que la mención al constructivismo es tratada en menor medida. Se puede afirmar que dichas definiciones, al ser generales, poseen un rasgo abierto, que permiten su empleo dentro de un enfoque constructivista; sin embargo, ese no es el caso en varios de los textos estudiados; por ejemplo, Purvis et al. (2012), quienes parten de un sentido integral de la sostenibilidad, claramente, enmarcan la dimensión económica dentro de la epistemología económica neoclásica, al sostener que la sostenibilidad se logra mediante la adopción de modelos productivos competitivos. Asimismo, autores que explícitamente no parten de esta epistemología, no se apartan completamente de ella, al conservar concepciones propias de la economía liberal; tal es el caso de Guzmán \& Alonso (2007), quienes entienden la sostenibilidad como explotación de los recursos naturales, que no arriesga su disponibilidad para las generaciones futuras, lo que reproduce el sentido de naturaleza, como recurso de la economía liberal. Igualmente, Martin \& Sauerborn (2013) definen la agricultura sostenible, como la articulación de las dimensiones ecológica, social, económica, mediante los conceptos de solidez ambiental, rentabilidad económica 
y equidad social, los cuales, de nuevo, se pueden interpretar como enmarcados dentro de una visión económica neoclásica del bienestar social y ambiental.

Por su parte, las posiciones críticas de la epistemología hegemónica, no exhiben un acuerdo alrededor de los aspectos o las dimensiones que se deben considerar para desarrollar un sentido de la sostenibilidad, realmente alternativo o radical; por ejemplo, el aspecto de género y la incorporación del feminismo o ecofeminismo dentro del discurso transdisciplinario de la agroecología en las dimensiones social y económica de la sostenibilidad integral es considerado fundamental por Papuccio (2007) y Siliprandi (2010), pero no es mencionado en la gran mayoría de los textos estudiados. Igualmente, Rech (2006), Gliessman (2007) y Cox (2014a; 2014b) realizan un particular énfasis en la necesidad de considerar la dimensión ética de la sostenibilidad, pero no es un aspecto estructural de las demás propuestas, aquí estudiadas. De la misma forma, Sans (2007) cambia las dimensiones habituales de la sostenibilidad integral, para las dimensiones social y económica, bajo las categorías de producción de alimentos inocuos, que comprende la calidad y la seguridad alimentaria y equipara el desarrollo rural a la viabilidad económica y social de la población rural.

Además, estas posiciones críticas no siempre adoptan la epistemología alternativa, establecida dentro de la teoría agroecológica. La sistémica, ha sido una de las bases epistemológicas de la agroecología, lo que significa, en el caso de la sostenibilidad, que sus componentes no deben ser analizados de manera aislada sino en función de las relaciones que establecen con el resto del proceso de sostenibilidad (Gomes, 2005; Muro, 2007; Borsatto \& Carmo, 2012); sin embargo, no todas las aproximaciones son sistémicas dentro de la teoría agroecológica; por ejemplo, Raza et al. (2012), se ocupan únicamente de la sostenibilidad del suelo y del recurso agua dentro de la dimensión ecológica, sin entrar a establecer las relaciones de estos componentes con las otras dimensiones o con los demás elementos dentro de la dimensión ecológica. Por su parte, Timmerman \& Félix (2015), se centran en el concepto de trabajo sostenible dentro de la dimensión económica, afirmando que es sostenible si logra mantener un mínimo de trabajos de calidad, particularmente, en el área rural, sin articular este término a otros elementos de la actividad económica o de las esferas social y ecológica.

Por otro lado, muchas de las propuestas de sostenibilidad dentro de la agroecología no parten de un enfoque constructivista, sino que, simplemente, no proporcionan una definición explícita de sostenibilidad ni tratan el asunto del universalismo de la epistemología convencional, por lo que dejan abierta la opción de una interpretación hegemónica de este término. Tal es el caso de Funes-Aguilar \& Monzote (2006), quienes adoptan el término de desarrollo sostenible para su propuesta de agricultura sostenible, pero no proporcionan una definición clara del primero, que permita dilucidar una epistemología alternativa, en su elaboración teórica. Igualmente, Pérez \& Soler (2013) y Lemanceau et al. (2015), se adhieren a la agricultura sostenible como fin de la agroecología, pero no proporcionan una definición; esto es un claro descuido teórico, pues no existe una definición canónica. Precisamente, Sarandón \& Flores (2009) advierten que al no existir un consenso es necesario un planteamiento explícito del marco de evaluación, en el que se establezca la definición de agricultura sostenible adoptada y los criterios que se consideran se deben cumplir, para lograr dicha sostenibilidad.

Sistema de referencia: Al igual que sucede con la definición de sostenibilidad, no existe un consenso en torno a cuál es el sistema de referencia fundamental de la agroecología. Esta divergencia, se puede agrupar en dos tendencias generales, en función de lo que se considera debe perseguir la ciencia agroecológica.

La primera tendencia, se centra en los sistemas de producción con seres vivos. El punto de partida es que éstos deben ser gestionados a partir de criterios basados en la ecología (Bello et al. 2010; Weiner et al. 2010; Gliessman, 2013); sin embargo, el enfoque se ha ido ampliando y hoy se puede hablar de tres niveles jerárquicos dentro de esta tendencia: el primer nivel es el agroecosistema o sistema de producción con seres vivos (Peireira et al. 2006; Parra, 2013; Lemanceau et al. 2015), que se ha definido, habitualmente, como el proceso de circulación de materia y energía, a través de un conjunto de organismos para la producción de biomasa (Sevilla, 2006; Gliessman, 2007; Martin \& Sauerborn 2013); no obstante, León (2014) señala que si la agroecología se va a enmarcar dentro de un pensamiento ambiental, el elemento social o cultural debe estar implícito dentro de la definición de agroecosistema; por esto, dicho autor propone una definición que tenga en cuenta las relaciones simbólicas, sociales, económicas y políticas, que se encuentran involucradas en los procesos de producción con seres vivos.

Dentro del enfoque de agroecosistemas, Gutiérrez et al. (2008) anotan que un agroecosistema se puede considerar sostenible cuando satisface unas metas productivas sin comprometer la organización de los sistemas, a partir de los cuales, genera su operación de distinción y sin depender de insumos ajenos a su entorno inmediato; además, agregan que un sistema en esta situación debe ser resiliente y adaptable. Con respecto a la dimensión social, dichos autores señalan que es sostenible, si su organización se puede mantener en el tiempo, mediante relaciones equitativas y que aporten a la reproducción social y biológica del grupo generador. Para alcanzar esto, Gutiérrez et al. (2008) apuntan que se deben buscar relaciones en el entorno social, que disminuyan prác- 
ticas de consumo excesivo y de generación de pobreza, las cuales, son ambiental y socialmente no sostenibles, respectivamente. De manera similar, Altieri (2009) sostiene que para lograr la sostenibilidad de los agroecosistemas es necesario realizar cambios estructurales, a nivel político, cultural, económico y social, en el entorno social; sin embargo, no es claro, en este autor, que propugne por un cambio profundo de la epistemología convencional, que conduzca a un sistema civilizatorio con esferas económicas, políticas y culturales, diferentes a las propias de la modernidad ortodoxa.

El segundo nivel de la tendencia productivista es la agricultura, donde se presentan dos tipos de definiciones de agricultura sostenible: una general y una específica; en el primer grupo, se encuentran Funes-Aguilar \& Monzote (2006), quienes escriben que la agricultura sostenible es aquella que busca generar una producción en el tiempo, a partir de la aplicación de la ecología a la producción agraria. Igualmente, Rocha \& Siman (2007) y Martin \& Sauerborn (2013) proporcionan una definición general, partiendo del concepto de desarrollo sostenible, para anotar que la agricultura sostenible es aquella forma de producción con seres vivos en la que se satisfacen las necesidades humanas presentes, sin comprometer la capacidad del sistema agrario, de satisfacer las necesidades de las generaciones futuras.

Por otro lado, se han dado definiciones más específicas de la agricultura sostenible; por ejemplo, Peireira et al. (2006), la definen como aquella centrada en cinco elementos: el sostenimiento a largo plazo de los recursos naturales y el rendimiento agrícola; mejor compatibilidad entre la agricultura y el potencial de los agroecosistemas; disminución del uso de insumos externos y fuentes de energía no renovables; generación de alimentos y renta adecuados y satisfacción de las necesidades sociales de la población rural. Por su parte Gliessman (2007) señala que la agricultura sostenible debe tener un bajo impacto ambiental negativo, asegurar la renovación del suelo, no agotar los cuerpos de agua, operar a partir de insumos locales, mantener la biodiversidad silvestre y la agrobiodiversidad, permitir el control local de los recursos agrícolas y generar procesos de acceso y de apropiación de conocimientos, prácticas y tecnologías agrícolas. Por su parte, Kershen (2012), se ciñe a la definición dada por la Ley Agraria de 1990, de Estados Unidos, que señala que la agricultura sostenible es aquella capaz de satisfacer los requerimientos humanos de recursos; mejorar las condiciones del entorno natural del cual depende la agricultura; hacer un uso óptimo, tanto de energía no renovable como de los recursos provenientes de la finca, al igual, que integra, cuando es pertinente, los ciclos y los controles naturales; hacer viable la economía de la finca y mejora la calidad de vida de los agricultores y de la sociedad como un todo.

Respecto a cómo lograr la sostenibilidad agraria, Cox (2014a) sostiene que es necesario un cambio en la mentalidad de las personas que, en el caso de la agricultura, implica una identificación de las personas con el territorio y los seres vivos que lo habitan. Esta conciencia debe conducir a una nueva visión del mundo, en el que no se de una escisión entre el ser humano y los demás seres vivos y, por lo tanto, se entienda que la sostenibilidad no es para los seres humanos sino para la biosfera. Además, Cox (2014b) apunta que un cambio hacia la agricultura sostenible debe abandonar la obsesión de la agricultura convencional por el rendimiento económico, al igual que debe incorporar otras metodologías y epistemologías, en las que se dé un papel explícito a los valores y a la espiritualidad, enmarcándose, claramente, en una epistemología distinta, a la de la modernidad hegemónica.

De manera similar, varios autores resaltan la importancia de generar una agricultura desde abajo, es decir, a partir del conocimiento local, unidades productivas familiares y en beneficio de las productoras. En este sentido, Guzmán \& Alonso (2007) anotan que un sistema agrario sostenible debe permitir la autonomía de los productores y productoras, la aceptabilidad cultural de las tecnologías y debe generar procesos equitativos de distribución de los beneficios. Por su parte, Rosset \& Martínez-Torres (2012) prefieren hablar agricultura campesina sostenible, que presenta, entre sus objetivos, la soberanía alimentaria en vez de la articulación de la producción agraria al mercado y anotan que ésta se logra a partir del ingenio, la innovación y el conocimiento de los campesinos.

Dentro de la tendencia centrada en la producción ha surgido un enfoque, desde el 2003, que sostiene que para alcanzar el objetivo de la sostenibilidad integral de la agricultura, se debe cambiar el foco de la sostenibilidad de los agroecosistemas a la sostenibilidad de los sistemas alimentarios, es decir, a todo el sistema de producción, de distribución y de consumo de alimentos (Gliessman, 2007; De Schutter, 2012; Borsatto \& Carmo, 2013; Calle et al. 2013). Aunque este enfoque pretende ser más comprensivo al afirmar que la producción no es sostenible si el resto de la red económica no es sostenible, tiene el problema de reducir la producción con seres vivos a la alimentación, lo que simplifica, considerablemente, los bienes y servicios que las poblaciones humanas obtienen de la biosfera.

Para lograr un sistema alimentario sostenible, varios autores escriben sobre la necesidad de cambiar los hábitos alimenticios, con el fin que los individuos y las poblaciones humanas adopten patrones de dieta sostenibles (Wezel \& David, 2012; Pérez \& Soler 2013). A su vez, otros autores mencionan que se deben estudiar y reconfigurar las relaciones de poder que existen, a través de todo el sistema alimentario, desde el campo de cultivo hasta la mesa. Para esto, es necesario crear políticas e instituciones que estén enfocadas a la implementación de sistemas alimentarios sostenibles, que 
involucran el aseguramiento de un buen nivel de vida de los productores, al igual que el derecho a la alimentación (De Schutter, 2012; González, 2012; Méndez et al. 2013).

La segunda tendencia sobre el tipo de sostenibilidad que debe perseguir la agroecología está representada por aquellas propuestas que hacen un énfasis mayor en las poblaciones rurales, cambiando el foco de la producción al desarrollo. Aquí, se hace una articulación más explícita las dimensiones social y económica de la sostenibilidad integral, planteando que, tanto la degradación ambiental como la pobreza, no son fenómenos sostenibles.

Dentro de esta tendencia, se plantean dos sistemas de referencia: el desarrollo sostenible y el desarrollo rural sostenible Ambos privilegian el sector rural, resaltando la necesidad de la participación campesina y su autonomía para lograr el objetivo del desarrollo sostenible, pero, el segundo, es más enfático, al establecer el desarrollo rural sostenible como el objetivo de la agroecología (Machado \& Carmo, 2007; Papuccio, 2007; Andrade \& Casali, 2011). Asimismo, la tendencia desarrollista hace un énfasis en la pobreza y en el hambre, por lo que conceptos, como soberanía y seguridad alimentaria, se vuelven centrales, dentro de su teoría de la sostenibilidad (Altieri, 2009; Ferguson et al. 2009; Sámano, 2013). Además, se habla de la necesidad de incorporar una agroecología política, en la que se encuentren herramientas jurídicas, institucionales y de organización social, que permitan implementar modelos de producción con seres vivos sostenibles (Garrido, 2012; González, 2012; Calle et al. 2013).

La mayoría de autores enmarcados en esta tendencia parten de una concepción constructivista del desarrollo, afirmando que debe ser un proceso particular de cada sociedad. De igual forma, agregan que un verdadero desarrollo se logra a través de su construcción participativa, en la que se privilegie la autonomía, los conocimientos locales y los sistemas de producción de tipo familiar, en el sector rural (Funes-Aguilar \& Monzote, 2006; Warner, 2008; Calle et al. 2013); sin embargo, esta resignificación del desarrollo no considera la carga epistemológica del concepto de desarrollo que, para algunos autores de otros campos del ecologismo, conduce a una reproducción de la epistemología de la economía y la política convencionales (Sachs, 1996; Latouche, 2003). De esto son conscientes Gutiérrez et al. (2008), quienes se oponen a la idea de desarrollo como concepto estructurador de la teoría agroecológica, aduciendo que es uno de los meta relatos de la modernidad, que no permite superarla, pues en el fondo sigue manteniendo las promesas de esta forma de vida, las cuales, son el control sobre la naturaleza, la abundancia material, las organizaciones políticas centralizadas eficientes y democráticas, la paz y la justicia y una cultura superior.
De la misma forma, se puede anotar que, a pesar del distanciamiento de esta propuesta de los discursos de la modernidad y de las ciencias convencionales, el desarrollismo no logra superar el antropocentrismo moderno, que también se evidencia en la gran mayoría de autores, de la tendencia productivista. Exceptuando Rech (2006) y Cox (2014a; 2014b), en los discursos de sostenibilidad de la agroecología, no hay estrategias que se ocupen de los seres vivos no humanos, como miembros de la comunidad ética, que esta ciencia pretende construir. Igualmente, a excepción de Papuccio (2007) y Siliprandi (2010), tampoco hay una mención de las relaciones de poder en torno al género dentro de la búsqueda de equidad, dentro de la dimensión social de la sostenibilidad integral.

Resiliencia: Al concepto de sostenibilidad, se la han otorgado diversos atributos, a través de su desarrollo teórico. Dentro de la agroecología, Guzmán \& Alonso (2007) recogen los planteados por el Marco para la Evaluación de Sistemas de Manejo de Recursos Naturales Incorporando Indicadores de Sostenibilidad MESMIS: productividad, estabilidad, resiliencia, equidad social, autonomía, y adaptabilidad cultural; por su parte, Gutiérrez et al. (2008) mencionan estabilidad, resiliencia, adaptabilidad y productividad, eficiencia y eficacia en la producción; González (2012) y González \& Caporal (2013) plantean los mismos del MESMIS, aunque no incluyen la adaptabilidad cultural. Aunque estos atributos son tratados en mayor o menor medida por la mayoría de textos consultados, la resiliencia merece especial interés, ya que Álvarez-Salas et al. (2014) proponen la resiliencia socioecológica de los agroecosistemas, como el objeto de estudio de la agroecología, argumentando que responde mejor a las problemáticas y a los fenómenos estudiados por la agroecología, al igual que a su objetivo de la sostenibilidad de los agroecosistemas, que los agroecosistemas en sí.

Si bien es cierto que la resiliencia se menciona principalmente en relación al cambio climático, es importante resaltar que la resiliencia socioecológica es un concepto diferente a resiliencia. Este último, se ha definido, habitualmente, como la capacidad de un sistema para mantener su organización frente a perturbaciones (Nicholls, 2013). Por el contrario, Ríos-Osorio et al. (2013b) definen la resiliencia socioecológica de los agroecosistemas, como la capacidad que tienen estos últimos para mantener su organización y unos niveles satisfactorios de producción, tras una perturbación sin poner en riesgo la organización de los sistemas sociales y biológi$\cos$, que constituyen su entorno.

Uno de los aspectos más llamativos de esta nueva propuesta es que hace más explícito el objetivo de la agroecología, pues muestra que no es una ciencia que tiene como fin el conocimiento y la comprensión de los agroecosistemas, sino la sostenibilidad de éstos y parte del supuesto que, estudiando su 
resiliencia socioecológica, se puede alcanzar más fácilmente el objetivo de la sostenibilidad de los agroecosistemas. Aunque son pocos los textos que se suscriben a este nuevo enfoque y todos se enmarcan dentro de la agroecología de agroecosistemas, la definición de resiliencia socioecológica es extrapolable a los otros sistemas de referencia existentes dentro de la agroecología y, claramente, no entra en pugna con su objetivo de la sostenibilidad integral.

En conclusión, el concepto de sostenibilidad en agroecología muestra las mismas dificultades epistemológicas que se dan en el marco general de los debates ecologistas y ambientalistas. Por un lado, existe la discusión de si es posible establecer una definición universal, objetivo del término. Aunque la agroecología, de manera general, está a favor de un enfoque constructivista, no siempre lograr superar el universalismo de la modernidad ortodoxa al reproducir discursos de ella, como la economía de mercado, el derecho, el desarrollo o la organización política alrededor de instituciones formales centralistas, como el Estado-Nación.

Por otro lado, la agroecología -a su interior- no ha podido generar un consenso alrededor de cuál es el sistema que se debe sostener. Esta indefinición, tanto del concepto de sostenibilidad como de su sistema de referencia, se puede interpretar como una cualidad del enfoque constructivista, propio de la agroecología, ya que permite cierta flexibilidad, al mismo tiempo, que rechaza, explícitamente, el reduccionismo y el universalismo, propios de la epistemología científica convencional, pero los textos estudiados muestran que, muchas veces, se da por una falta de diálogo entre las distintas propuestas teóricas o por una carencia de rigor teórico, a la hora de abordar el concepto de sostenibilidad; no obstante, existen puntos de convergencia dentro de las distintas propuestas teóricas agroecológicas. En primer lugar, existe un interés general en darle cabida a discursos marginales, como el conocimiento tradicional y los saberes populares; segundo, se presenta un consenso, en que la agroecología no puede ser universalista y, por lo tanto, debe haber apertura en sus planteamientos teóricos, como es el de la sostenibilidad; sin embargo, se reconoce la necesidad de unos mínimos, rechazando el relativismo ingenuo del todo, vale a favor de propuestas, donde el bienestar de las poblaciones rurales y la sostenibilidad ecológica sean criterios que tienen que estar incluidos en cualquier sentido de sostenibilidad, elaborado al interior de la ciencia agroecológica; en tercer lugar, existe un consenso en que la agroecología y su concepto de sostenibilidad deben partir de concepciones no reduccionistas y disciplinares de estos campos, por lo que se ha hablado de enfoques, como el sistémico y la transdisciplinariedad y, por último, es claro que la agroecología todavía necesita avanzar en la construcción de una postura autorreflexiva, donde haya una constante revisión de los discursos teóricos que se van construyendo, con el fin de alcanzar un trasfondo común, que permita evaluar su validez y su consistencia, como práctica científica racional. Esto parece reproducir la epistemología de la ciencia convencional, pero mientras que no se propongan medios alternativos para evaluar estos rasgos, la agroecología debe seguir aceptando ciertos elementos de la imagen heredada de la ciencia.

Conflicto de intereses: El manuscrito fue preparado y revisado con la participación de todos los autores, quienes declaramos que no existe conflicto de intereses que ponga en riesgo la validez de los resultados presentados.

\section{BIBLIOGRAFÍA}

1. ALTIERI, M.A. 2009. Escalonando la propuesta agroecológica para la soberanía alimentaria. Agroecología. (España). 4:39-48.

2. ÁlVAREZ-SALAS, L.M.; POLANCO-ECHEVERRY, D.N.; RÍOS-OSORIO, L.A. 2014. Reflexiones acerca de los aspectos epistemológicos de la agroecología. Cuad. Desarro. Rural. (Colombia). 11:55-74.

3. ANDRADE, F.M.C.; CASALI, V.W.D. 2011. Homeopatia, agroecologia e sustentabilidade. Rev. Bras. Agroecologia. 6(1):49-56.

4. BELLO, A.; JORDÁ, C.; TELLO, J.C. 2010. Agroecología y producción ecológica. Catarata. (Madrid). 77p.

5. BORSATTO, R.S.; CARMO, M.S. 2012. Agroecologia e sua epistemologia. Interciencia. (Venezuela). 37(9):711-716.

6. BORSATTO, R.S.; CARMO, M.S. 2013. A Agroecologia como um campo científico. Rev. Bras. Agroecología 8:4-13.

7. CALLE, A.; GALLAR, D.; CANDÓN, J. 2013. Agroecología política: la transición social hacia sistemas agroalimentarios. Rev. Econ. Crítica. (España). 16:244277.

8. COX, T.E.B. 2014a. Grafting metaphysics: how transpersonal agroecology bear fruit with process metaphysics as its roots. Process Stud. (Estados Unidos). 43(2):104-128.

9. COX, T.E.B. 2014b. Transpersonal Agroecology: the metaphysics of alternative agricultural theory. J. Transpersonal Psychol. (Estados Unidos). 46(1):35-57. 
10. DE SCHUTTER, O. 2012. Agroecology, a tool for the realization of the right to food. En: Lichtfouse, E. (ed). Agroecology and strategies for climate change. Springer (Dordrecht). p.1-16.

11. DILWORTH, C. 2009. General principles. En: Boersema, J.J.; Reijnders, L. (eds). Principles of environmental sciences. Springer Science/Business Media B.V. (Dordrecht). p.75-83.

12. FERGUSON, B.G.; MORALES, H.; GONZÁLEZ, A.; ÍNIIGUEZ, F. de J.; MARTÍNEZ, M.E.; McAFEE, K.; NIGH, R.; PERFECTO, I.; PHILPOTT, S.M.; SOTO, L. 2009. La soberanía alimentaria: cultivando nuevas alianzas entre campo, bosque y ciudad. Agroecología. 4:49-58.

13. FUNES-AGUILAR, F.; MONZOTE, M. 2006. Sistemas agroecológicos y su papel en los países del Tercer Mundo. Av. Invest. Agropec. (México). 10(3):5-27.

14. GARRIDO, F. 2012. Ecología política y agroecología: marcos cognitivos y diseño institucional. Agroecología. 6:21-28.

15. GLIESSMAN, S.R. 2007. Agroecology. The ecology of sustainable food systems. CRS Press. (Boca Raton). 384p.

16. GLIESSMAN, S.R. 2013. Agroecología: plantando raíces de la resistencia. Agroecología. 8(2):19-26.

17. GOMES, J.C.C. 2005. Bases epistemológicas da agroecologia. En: Aquino, A.M.; Assis, R.L. (eds). Agroecologia. Princípios e técnicas para uma agricultura orgánica sustentável. Embrapa Informação Tecnológica (Brasília, DF). p.71-99.

18. GONZÁLEZ, M. 2012. Algunas notas sobre agroecología y política. Agroecología. 6:9-21.

19. GONZÁLEZ, M.; CAPORAL, F.R. 2013. Agroecología y política. ¿Cómo conseguir la sustentabilidad? Sobre la necesidad de una agroecología política. Agroecología. 8(2):35-43.

20. GUTIÉRREZ, J.G.; AGUILERA, L.I.; GONZÁLEZ, C.E. 2008. Agroecología y sustentabilidad. Convergencia. (México). 46:51-87.

21. GUZMÁN, G.I.; ALONSO, A.M. 2007. La investigación participativa en agroecología: una herramienta para el desarrollo sustentable. Ecosistemas. (España). 16(1):24-36.
22. KERSHEN, D.L. 2012. The contested vision for agriculture's future: Sustainable Intensive Agriculture and Agroecology. Creighton Law Rev. (Estados Unidos). 46:591-618.

23. LATOUCHE, S. 2003. Decrecimiento y posdesarrollo. El pensamiento creativo contra la economía del absurdo. El Viejo Topo. (España). 150p.

24. LEMANCEAU, P.; MARON, P.A.; MAZURIER, S.; MOUGEL, C.; PIVATO, B.; PLASSART, P.; RANJARD, L.; REVELLIN; C.; TANDY, V.; WIPF, D. 2015. Understanding and managing soil biodiversity: a major challenge in agroecology. Agron. Sustain. Dev. (Francia). 35(1):67-81.

25. LEÓN, T. 2014. Perspectiva ambiental de la agroecología. La ciencia de los agroecosistemas. Universidad Nacional de Colombia. (Bogotá). 398p.

26. LUFFIEGO, M.; RABADÁN, J.M. 2000. La evolución del concepto de sostenibilidad y su introducción en la enseñanza. Enseñan. Cienc. (España). 18(3):473486.

27. MACHADO, R.; CARMO, M.S. 2007. A Agroecologia na construção do desenvolvimento rural sustentável. Rev. Bras. Agroecologia. 2(1):511-514.

28. MARTIN, K.; SAUERBORN, J. 2013. Agroecology. Springer Science/Business Media B.V. (Dordrecht). 330p.

29. MÉNDEZ, V.E.; BACON, C.M.; COHEN, R. 2013. La agroecología como un enfoque transdisciplinar, participativo y orientado a la acción. Agroecología. 8(2):9-18.

30. MURO, P. 2007. La investigación en agroecología: transdisciplinariedad y multidimensionalidad. Rev. Bras. Agroecologia. 2(1):570-575.

31. NICHOLLS, C.I. 2013. Enfoques agroecológicos para incrementar la resiliencia de los sistemas agrícolas al cambio climático. En: Nicholls, C.I.; Ríos-Osorio, L.A.; Altieri, M.A. (eds). Agroecología y resiliencia socioecológica: adaptándose al cambio climático. CYTED. (Medellín). p.18-29.

32. PAPUICCIO, S. 2007. El enfoque de género en la agroecología: un aporte clave para alcanzar la sustentabilidad. Rev. Bras. Agroecologia. 2(1):1649-1653.

33. PARRA, R. 2013. La agroecología como un modelo económico alternativo para la producción sostenible 
de alimentos. Orinoco Pensam. Prax. (Venezuela). 1(3):24-36.

34. PEIREIRA, L.C.; SILVEIRA, M.A. da; NETO, F.L. 2006. Agroecologia e aptidão agrícola das terras: as bases científicas para uma agricultura sustentável. Rev. Bras. Agroecologia. 1(1):823-826.

35. PÉREZ, D.; SOLER, M. 2013. Agroecología y ecofeminismo para decolonizar y despatriarcalizar la alimentación globalizada. Rev. Int. Pensam. Polít. (España). 8:95-113.

36. PURVIS, G.; DOWNEY, L.; BEEVER, D.; DOHERTY, M.L.; MORAHAN, F.J.; SHERIDAN, H.; McMAHON, B.J. 2012. Development of a sustainably-competitive agriculture. En: Lichtfouse, E. (ed). Agroecology and strategies for climate change. Springer. (Dordrecht). p.35-65.

37. RAZA, A.; FRIEDEL, J.K.; BODNER, G. 2012. Improving water use efficiency for sustainable agricultura. En: Lichtfouse, E. (ed). Agroecology and strategies for climate change. Springer. (Dordrecht). p.167-211.

38. RECH, C.M. 2006. Ecosofia: um principio elementar à Agroecologia. Rev. Bras. Agroecologia. 1(1):13991400 .

39. RÍOS-OSORIO, L.A.; CRUZ-BARREIRO, I.C.; WELSHRODRÍGUEZ, C.M. 2013a. The concept of sustainable development from an ecosystem perspective: history, evolution, and epistemology. En: Yañez-Arancibia, A.; Dávalos, R.; Day, J.W.; Reyes, E. (eds). Ecological dimensions for sustainable development. WIT Press. (Southampton). p.29-45.

40. RÍOS-OSORIO, L.A.; SALAS-ZAPATA, W.; ESPINOSAALZATE, J.A. 2013b. Resiliencia socioecológica de los agroecosistemas. Más que una externalidad. En: Nicholls, C.I.; Ríos-Osorio, L.A.; Altieri, M.A. (eds). Agroecología y resiliencia socioecológica: adaptándose al cambio climático. CYTED. (Medellín). p.6076.

41. ROCHA, J.M.; SIMAN, R.F. 2007. Agroecologia: um contraponto à produtividade insustentável da agricultura convencional. Rev. Bras. Agroecologia. 2(1):29-32.

42. ROSSET, P.; MARTÍNEZ-TORRES, M.E. 2012. Rural social movements and agrecology: context, theory, and process. Ecol. Soc. (Canada). 17(3):17. Disponible desde Internet en: http://dx.doi.org/10.5751/ES05000-17031747.
43. SÁMANO, M.A. 2013. La agroecología como una alternativa de seguridad alimentaria para las comunidades indígenas. Rev. Mex. Cienc. Agríc. 4(8):19-28.

44. SACHS, W. (ed.). 1996. Diccionario del desarrollo. Una guía del conocimiento como poder. PRATEC. (Perú). 399p.

45. SANS, F.X. 2007. Agroecología. Ecosistemas. (España). $16(1): 1-2$

46. SARANDÓN, S.J.; FLORES, C.C. 2009. Evaluación de la sustentabilidad en agroecosistemas. Agroecología. 4:19-28.

47. SEVILLA, E. 2006. De la sociología rural a la agroecología. Icaria. (Barcelona). 255p.

48. SIKOR, T.O.; NORGAARD, R.B. 1999. Principles for sustainability: protection, investment, cooperation, and innovation. En: Köhn, J. (ed). Sustainability in question. The search for a conceptual framework. Edward Elgar. (Cheltenham). p.49-65.

49. SILIPRANDI, E. 2010. Mujeres y agroecología. Nuevos sujetos políticos en la agricultura familiar. Invest. Fem. (España). 1:125-137.

50. SILVA, R.D.; DE BIASE, L. 2012. Na encruzilhada dos saberes e práticas: inserções antropológicas sobre estranhamento e alteridade no interior da Agroecologia. Rev. Bras. Agroecologia. 7(2):3-18.

51. TIMMERMANN, C.; FÉLIX, G. 2015. Agroecology as a vehicle for contributive justice. Agric. Hum. Values (Países Bajos). Disponible desde Internet en: http://link.springer.com/article/10.1007\%2 Fs10460-014-9581-8. Doi: 10.1007/s10460-0149581-8

52. TOMICH, T.P.; BRODT, S.; FERRIS, H.; GALT, R.; HORWARTH, W.R.; KEBREAB, E.; LEVEAU; J.H.J.; LIPTZIN, D.; LUBELL, M.; MEREL, P.; MICHELMORE, R., ROSENSTOCK, T.; SCOW, K.; SIX, J.; WILLIAMS, N.; YANG, L. 2011. Agroecology: a review from a global-change perspective. Ann. Rev. Environ. Resour. (Estados Unidos). 36:193-222.

53. WARNER, K.D. 2008. Agroecology as participatory science: emerging alternatives to technology transfer extension practice. Sci. Technol. Hum. Values. (Estados Unidos). 33(6):754-777. 
54. WEINER, J.; ANDERSEN, S.B.; WIBKE, K.-M. W.; GRIEPENTROG, H.W.; OLSEN, J.M. 2010. Evolutionary agroecology: the potential for cooperative, high density, weed-suppressing cereals. Evol. Appl. (Estados Unidos). 3:473-479.

55. WEZEL, A.; DAVID, C. 2012. Agroecology and the food system. En: Lichtfouse, E. (ed). Agroecology and strategies for climate change. Springer. (Dordrecht). p.17-33.
56. WEZEL, A.; SOLDAT, V. 2009. A quantitative and qualitative historical analysis of the scientific discipline of agroecology. Int. J. Agric. Sustain. (Reino Unido). 7:3-18.

Recibido: Marzo 31 de 2015

Aceptado: Octubre 13 de 2015

Cómo citar:

Gómez, L.F.; Ríos-Osorio, L.A.; Eschenhagen Durán, M.L. 2015. El concepto de sostenibilidad en agroecología. Rev. U.D.C.A Act. \& Div. Cient. 18(2): 329-337. 